\title{
1.2 DYNAMICS OF THE CRAB NEBULA
}

\author{
VIRGINIA TRIMBLE*
}

Institute of Theoretical Astronomy, University of Cambridge, Cambridge, U.K.

\begin{abstract}
Studies of the proper motion of many filaments in the Crab Nebula show that the expansion centre does not coincide with the present position of the pulsar NP 0532. Possible reasons for this difference are discussed. Estimates of the turbulent velocity within the nebula indicate that it lies in the range 100 to $300 \mathrm{~km} \mathrm{sec}^{-1}$. An analysis of the present expansion rate of the nebula indicates a convergence at $1140 \pm 10 \mathrm{AD}$. The acceleration implied in this result could arise from magnetic or relativistic electron pressure.
\end{abstract}

\section{Introduction}

Proper motions of features in the Crab Nebula have been reported by Duncan (1921, 1939), Deutsch and Lavdovsky (1940) and Trimble (1968). Radial velocities have been given by Sanford (1919), Mayall (1937, 1962), Woltjer (1958), Münch (1958), Trimble (1968), and Münch et al. (1971). Several kinds of information can be derived from this dynamical data. The distance to the nebula is almost certainly in the range $1030 \mathrm{pc}$ (Woltjer, 1958) to $2170 \mathrm{pc}$ (Trimble, 1970a), with the most probable value lying near the middle of the range (Woltjer, 1970). The kinetic energy of the expanding remnant is, to within a factor of two, $10^{49} M / M_{\odot}$ ergs, but the value of the mass may be anywhere in the range one (Minkowski, 1968) to 10 (Gott et al., 1970) solar masses. Limits to rotational and turbulent energy will be set below.

\section{The Space Motion of the Nebula and NP 0531}

The center of expansion of the Crab Nebula and the time scale of that expansion (in the sense of distance covered divided by present velocity) are two of the better determined properties of the object. This results from the large number of individual proper motions of filaments which have been measured. It is, therefore, significant that the expansion center does not coincide with the present position of the central star and that the time scale does not agree with the known age of the supernova remnant.

The position of the expansion center, determined by the intersection of the filamentary proper motion vectors (without regard to time) allows us to determine the proper motion of the central star and the nebula separately. It is evident that, in 1054, all the material must have been at the expansion center (provided only that supernovae occur in objects of more or less stellar dimensions), and that the star (NP 0531, Baade's Star, Star E of Trimble, 1968) is now where it is. Its proper motion is, therefore, just that change in position divided by the time elapsed since the Chinese saw their 'guest star'. The motion so found is

$$
\mu_{\alpha}=-0.0116 \pm 0.0022^{\prime \prime} / \mathrm{yr} \text { and } \mu_{\delta}=0.0048 \pm 0.0022^{\prime \prime} / \mathrm{yr}
$$

\footnotetext{
* NATO Postdoctoral Research Fellow.
} 
where the uncertainties come largely from the 3 " uncertainty in the position of the expansion center (Trimble, 1968). This is in reasonable accord with directly measured values of the stellar proper motion (Minkowski, 1970).

The proper motion of the nebula can be found by analyzing asymmetries in the filamentary proper motions around the expansion center, where the center is determined from purely geometric considerations and not by requiring all the filaments to have been there at the same time. If, for example, the nebula were moving along its major axis, then $\mu-r /($ apparent age) ought to be systematically positive for filaments at one end of the axis and negative for those at the other (where $r$ is the present distance of a filament from the expansion center). The proper motion data for individual filaments imply in this way:

$$
\mu_{\alpha}=0.0000 \pm 0.0007^{\prime \prime} / \mathrm{yr} \text { and } \mu_{\delta}=-0.0016 \pm 0.0007^{\prime \prime} / \mathrm{yr}
$$

where the uncertainties are standard deviations found in a least squares solution for $\mu$ with all filaments weighted equally.

The motion of the system, nebula plus pulsar, is just the sum of (1) and (2), weighted by their respective masses, and is large only if most of the mass is in the star. This is shown in Table I.

\section{TABLE I}

Proper motions and space velocities of the NP 0531-Crab Nebula system for various values of $W=M_{0531} / M_{\mathrm{CN}}$. The system is assumed to be $2000 \mathrm{pc}$ from us.

\begin{tabular}{lllllll}
\hline$W$ & $\mu_{\alpha}$ & $\mu_{\delta}$ & $V_{r}$ & $\pi$ & $\theta$ & $z$ \\
\hline 0 & $-0.0000^{\prime \prime} / \mathrm{yr}$ & $-0.0016^{\prime \prime} / \mathrm{yr}$ & $0 \mathrm{~km} / \mathrm{sec}$ & $0 \mathrm{~km} / \mathrm{sec}$ & $-13 \mathrm{~km} / \mathrm{sec}$ & $-7 \mathrm{~km} / \mathrm{sec}$ \\
0 & & & +100 & +99 & -6 & -17 \\
0.2 & -0.0018 & -0.0005 & 0 & -1 & +4 & -17 \\
0.2 & & & +100 & +98 & +11 & -27 \\
0.5 & -0.0039 & +0.0005 & 0 & -4 & +21 & -31 \\
0.5 & & & +100 & +95 & +28 & -41 \\
1.0 & -0.0058 & +0.0016 & 0 & -6 & +39 & -42 \\
1.0 & & & +100 & +93 & +46 & -52 \\
2.0 & -0.0077 & +0.0027 & 0 & -9 & +66 & -54 \\
2.0 & & & +100 & +90 & +73 & -64 \\
4.0 & -0.0093 & +0.0035 & 0 & -11 & +71 & -63 \\
4.0 & & & +100 & +88 & +78 & -73 \\
$\infty$ & -0.0116 & +0.0048 & 0 & -13 & +92 & -77 \\
$\infty$ & & & +100 & +86 & +99 & -87
\end{tabular}

It is not possible to determine the radial velocity of NP 0531 because its optical spectrum is featureless (Lynds et al., 1969). For the nebula itself, a radial velocity of $-5.5 \mathrm{~km} / \mathrm{sec}$ was suggested by Trimble (1968) predicated on some not-very-plausible assumptions. A direct measurement could be made, for example, using a diaphragm in the shape of an elliptical annulus, which, when placed in the focal plane of the telescope, would admit to the spectrograph only light from the edges of the nebula. This has not been done. The mean radial velocity of the $10 \%$ of the 418 features for 
which measurements are available nearest to the edge of the nebula is $+66 \mathrm{~km} / \mathrm{sec}$. This is not necessarily indicative of what the result of the recommended operation might be.

Table I shows $\mu_{\alpha}, \mu_{\delta}$, and the space motion in galactic rotation coordinates of the nebula-pulsar system for various values of $W=M_{0531} / M_{\mathrm{CN}}$ if their common radial velocity is 0 or $+100 \mathrm{~km} / \mathrm{sec}$. For all reasonable values of $W$, the kinetic energy carried by star and nebula due to these motions will be at most $10 \%$ of the kinetic energy of the nebular expansion. Some unreasonable values of $W$ are included in the table as being appropriate to other possible interpretations of what the two measured values may mean. All values of $\mu$ are uncertain by about $0.003^{\prime \prime} / \mathrm{yr}$, producing a $30 \mathrm{~km} / \mathrm{sec}$ uncertainty in the space velocity, even if the radial velocity were well known.

Let us attempt for a moment that 'suspension of disbelief' so necessary for the appreciation of any work of fiction and inquire what, if anything, the numbers in the table are good for.

If both $W$ and $V_{r}$ are small, the object does not deviate greatly from circular motion. Galactic rotation $2 \mathrm{kpc}$ further out is only about $12 \mathrm{~km} / \mathrm{sec}$ slower than it is aur position (Schmidt, 1966). If $W$ is large, on the other hand, the object is unusual in preceding the galactic rotation at its position by more than the $65 \mathrm{~km} / \mathrm{sec}$ normally permitted (Mihalas and Routly, 1968).

A similar result, differently obtained, has prompted the suggestion by Woolf (cited by Gott et al., 1970) that the star which became SN 1054 was a run-away star from the I Geminorum association. As this association has coordinates $\alpha=6^{\mathrm{h}} 8^{\mathrm{m}}, \delta=23^{\circ} 31^{\prime}$ and distance $=1400 \mathrm{pc}$ (diameter $\sim 5^{\circ}=120 \mathrm{pc}$ ), the suggestion is, at first sight, a very attractive one, given the range of possible proper motions and radial velocities for the pulsar-nebula system.

The catch is as follows: Known run-away stars are massive objects (Blaauw, 1961). If SN 1054 was a massive star, then, given an upper limit to stable neutron star masses of at most $2 M_{\odot}$ or so, most of the mass must be in the nebula. That is, if the original star had a mass $12 M_{\odot}, W \leqslant 0.2$. And for small values of $W$, the space motion is small enough that no particular explanation seems to be required.

The run-away hypothesis for pulsars in general and NP 0531 in particular is further discussed elsewhere in this volume.

\section{Rotation and Turbulence in the Nebula}

No rotation about any axis is detectable in the Crab Nebula to within the uncertainty of the determination. This is not surprising since, given conservation of angular momentum, even one $\mathrm{km} / \mathrm{sec}$ of rotational velocity at the edge of the present nebula would correspond to a presupernova star which was rotationally unbound. The most stringent observational limit can be set to rotation about an axis parallel to our line of sight. An analysis of the deviations in position angle of the individual filamentary proper motion vectors from their corresponding radius vectors indicates that the nebula (as viewed from its own center) is rotating at a rate of $2.7 \pm 2.7^{\prime \prime} / \mathrm{yr}$. This 
corresponds to rotation energy of at most $8 \times 10^{45}\left(M / M_{\odot}\right)$ erg. For other possible rotation axes, the limits are about ten times larger.

There is, on the other hand, definite evidence for turbulence, that is, for random deviations from the general rule that the velocity vector of a filament is proportional to its radius vector. Some estimate of these deviations can be obtained from measured radial velocities. It is, of course, not possible to say in general whether the radial velocity of a given filament is precisely appropriate to its position along the line of sight or not, because that position can only be determined as the product of the velocity and the age of the nebula. On the other hand, since, in the plane of the sky, the filaments are confined so closely to an ellipse, it is reasonable to assume that, in three dimensions, they are confined within the ellipsoidal surface:

$$
\frac{x^{2}}{a^{2}}+\frac{y^{2}}{b^{2}}+\frac{z^{2}}{b^{2}}=1
$$

where $x$ and $y$ are coordinates along the major and minor axes in the plane of the sky, $z$ is the coordinate along the line of sight and $a$ and $b$ are the semi-major and semi-minor axes $\left(5.4\right.$ and $3.6 \times 10^{18} \mathrm{~cm}$ for a distance of $\left.2 \mathrm{kpc}\right)$. Thus the largest $z$ a given filament should have can be found from its $x$ and $y$ positions. Any velocity excess, $\Delta V$, given by

$$
\Delta V=\left|V_{r}^{\text {observed }}-\frac{z}{\text { age }}\right|
$$

must then be of the nature of a turbulent velocity, except that it will be only a lower limit since we have only an upper limit to $z$. Such a velocity excess is found for 37 of the 418 features for which radial velocities are available. (This excludes the features for which Mayall, 1962, gave only approximate velocities.) The mean value of the excesses is $102 \mathrm{~km} / \mathrm{sec}$.

An upper limit to the average turbulent velocity can be obtained from the proper motion data by comparing apparent deviations from purely radial motion with the uncertainties of the measurements. The quantities to be compared are the difference, $D_{1}$, of the proper motions as determined from two separate sets of measurements (of plates taken on the $100^{\prime \prime}$ and $200^{\prime \prime}$ telescopes),

$$
D_{1}=\mu\left(100^{\prime \prime}\right)-\mu\left(200^{\prime \prime}\right)
$$

for each filament and the difference, $D_{2}$, between the average of the two measured $\mu$ 's and the present radius vector divided by age,

$$
D_{2}=\bar{\mu}-r / t,
$$

for each filament. Histograms of these two quantities show the same means and dispersions and are virtually indistinguishable. Their striking similarity may be taken to indicate that a major fraction of the apparent deviation from uniform radial expansion is due to measuring errors.

The deviations from radial motion in the plane of the sky, therefore, provide only 
an upper limit to the turbulent velocity of the filaments. The mean, median, and rms values of

$$
V_{\mathrm{dev}}=\left[\left(\mu_{x}-x / t\right)^{2}+\left(\mu_{y}-y / t\right)^{2}\right]^{1 / 2}
$$

all fall in the range 0.014 to $0.024^{\prime \prime} / \mathrm{yr}$. This corresponds to at most $225 \mathrm{~km} / \mathrm{sec}$, assuming the nebula to be at a distance of $2 \mathrm{kpc}$, or $275 \mathrm{~km} / \mathrm{sec}$, correcting for projection effects.

The lower and upper limits found from radial velocities and proper motions respectively thus indicate that turbulence must be in the range 100 to $300 \mathrm{~km} / \mathrm{sec}$. This corresponds to an energy of $1-9 \times 10^{47} \mathrm{M} / \mathrm{M}_{\odot} \mathrm{erg}$, that is, at most, about $10 \%$ of the expansion energy.

\section{Acceleration of the Expansion}

By extrapolating the measured proper motions backward in time, it is possible to find the time as well as the place at which they best converge. This was first done by Baade (1942), using Duncan's (1939) data. He found the rather surprising result that convergence occurred not in 1054 (as it would if the motions had been constant over the lifetime of the Crab Nebula) or earlier (as it would if the motions had been slowed by interaction with the interstellar medium), but later than 1054, implying that the present speeds are larger than the average ones over the nebular lifetime. Baade was not altogether convinced of the reality of this acceleration and, aside from pointing out that it could not be due to radiation pressure from any reasonable central star, deferred consideration of it until it should be confirmed by more accurate proper motion measurements.

His result was indeed confirmed. Convergence occurs in $1140 \pm 10 \mathrm{AD}$, where the uncertainty is derived from the discrepancy of values obtained from proper motions measured independently on $100^{\prime \prime}$ and $200^{\prime \prime}$ direct photographs. If this is interpreted as meaning that there has been a constant acceleration over the history of the nebula, then the acceleration amounts to $0.0014 \mathrm{~cm} / \mathrm{sec}^{2}$ and the initial expansion velocity was $1700 \mathrm{~km} / \mathrm{sec}$ along the major axis and $1100 \mathrm{~km} / \mathrm{sec}^{2}$ along the minor axis. The energy input required to maintain such an acceleration is $2.5 \times 10^{38} M / M_{\odot} \mathrm{erg} / \mathrm{sec}$. This is of the same order as the electromagnetic radiation output of the object at all frequencies and the energy required to sweep up interstellar matter as the nebula expands, $8 \times 10^{38} N_{\mathrm{H}} \mathrm{erg} / \mathrm{sec}$, where $N_{\mathrm{H}}$ is the local density of the interstellar medium. This average acceleration will indeed be imparted to the nebula by outward pressure of a magnetic field of average strength $5 \times 10^{-4}\left(M / M_{\odot}\right)^{1 / 2} \mathrm{G}$ or relativistic particles of total energy $3 \times 10^{48} \mathrm{M} / \mathrm{M}_{\odot}$ erg. These are very nearly equal to the minimum field strength and particle energy required to produce the observed synchrotron radiation (Woltjer, 1958), if $M \sim 1$.

There is, however, no particular reason to expect the acceleration to have been constant. On the one hand, the larger the nebula gets, the more interstellar matter it has to deal with per unit time, while ambient magnetic field and relativistic particles lose energy adiabatically in the course of the expansion, and so one might expect 
the acceleration to decrease with time. On the other hand, if the central neutron star is providing a continuing input of relativistic particles, their pressure will increase with time (because most of the energy is in electrons whose synchrotron lifetimes are long compared to the age of the nebula and, perhaps, protons), and so one might expect the acceleration to increase with time. It is, therefore, probable that the acceleration is some complicated function of time.

The observations do not help very much to define this function. It is possible, though, to set an upper limit to the acceleration going on now which is low enough to be of some interest. Comparison of proper motions determined over various stretches of the available baseline (1939-1966) indicates that the present acceleration is surely not more than three times the average value mentioned above. This corresponds to an upper limit on the magnetic field plus relativistic particle energy of $2 \times 10^{49} M / M_{\odot}$ erg. Now of the particles injected over the history of the nebula, all those with synchrotron lifetimes greater than 1000 years will still be there, aside from having lost energy adiabatically to the expansion. This means that, at most, about $3 \times 10^{49} M / M_{\odot}$ erg (present particle energy plus kinetic energy of the expansion) can have ever been injected into the nebula in the form of relativistic protons. The resulting constraints upon pulsar models are discussed by Trimble and Rees (1970). If the mass of the nebula is significantly greater than one solar mass, the limits are not, however, so stringent as they suggest.

\section{The Mass of the Crab Nebula}

It is clear that the size of a variety of quantities discussed here depends critically upon the mass in the Crab Nebula. The amount of material producing the optical emission lines has long been believed to be at most about one solar mass (Minkowski, 1968 and references cited therein). And the space between the filaments must contain much less material than this to prevent the dispersion measure of NP 0531 changing as the nebula expands (Drake, 1969). It has, however, recently been suggested that, in addition to the ionized material which produces most of the emission lines, the filaments might also contain large amounts of neutral material at their centers (Davidson and Tucker, 1970), increasing the total nebular mass to as much as $10 M_{\odot}$ (Gott et al., 1970).

Some indication of the quantity of neutral material present can be obtained from the intensity of the $\lambda 6300$ radiation of [OI]. This radiation is necessarily produced in regions where hydrogen is neutral (and, therefore, does not contribute significantly to the intensity of $\mathrm{H} \beta$, from which the mass of ionized material is obtained). This is a result of the large cross-section for the charge exchange reaction

$$
\mathrm{H}^{+}+\mathrm{O}^{\circ} \rightarrow \mathrm{H}^{\circ}+\mathrm{O}^{+}
$$

which arises from the near identity of the ionization potentials of the two elements.

Unfortunately, neutral material has not yet had time to come into equilibrium with the synchrotron radiation field of the nebula in its 1000 year lifetime. The run of 
temperature through this part of the gas cannot, therefore, be calculated by the methods of Davidson and Tucker (1970). The temperature rather reflects cooling which occurred early in the nebular expansion and may thus be very low. One can easily calculate the amount of material at a given $T_{e}$ required to produce the observed $\lambda 6300$ intensity:

$$
I([\mathrm{OI}]) \simeq I(H \beta)=1.24 \times 10^{-11} \mathrm{erg} \mathrm{cm}^{-2} \mathrm{sec}^{-1}
$$

(O'Dell, 1962). This has been done by Trimble (1970b). Table II shows the amount of matter required to produce $\lambda 6300$ as a function of $T_{e}$. The abundances assumed are $N_{\mathrm{He}}=N_{\mathrm{H}} ; N_{\mathrm{O}}=6 \times 10^{-4} N_{\mathrm{H}}$ (Davidson and Tucker, 1970). The distance to the nebula was taken to be $2 \mathrm{kpc}$. If it is really only $1.5 \mathrm{kpc}$, then the tabulated amount of

\section{TABLE II}

Amount of neutral gas in the filaments of the Crab Nebula required to produce the observed intensity of [Or] $\lambda 6300$

\begin{tabular}{rl}
\hline$T_{e}(\mathrm{~K})$ & $\begin{array}{l}\text { Mass } \\
\text { (solar masses) }\end{array}$ \\
& \\
$10^{4}$ & 2.6 \\
$9 \times 10^{3}$ & 3.5 \\
$8 \times 10^{3}$ & 5.0 \\
$7 \times 10^{3}$ & 9.0 \\
$6 \times 10^{3}$ & 13 \\
$5 \times 10^{3}$ & 26 \\
$4 \times 10^{3}$ & 100 \\
\hline
\end{tabular}

matter will produce $I(\lambda 6300) \simeq 1.8 I(\mathrm{H} \beta)$. It is, therefore, by no means unlikely that the nebular mass is significantly larger than has usually been assumed, and it is, in any case, very uncertain. The amounts of energy in various forms which must be supplied to the nebula during and after the supernova event are thus uncertain by factors of about 10. The kinetic energy of the expansion, for instance, may be in excess of $10^{50} \mathrm{erg}$ !

Trimble and Woltjer (1971) have recently presented a dynamical argument for the nebula mass not being much larger than $1 M_{\odot}$.

\section{References}

Baade, W.: 1942, Astrophys. J. 96, 109.

Blaauw, A.: 1961, Bull. Astron. Inst. Neth. 15, 265.

Davidson, K. and Tucker, W.: 1970, Astrophys. J. 161, 437.

Deutsch, A. N. and Lavdovsky, V. V.: 1940, Pulkovo Obs. Circ. 30, 21.

Drake, F. D.: 1969, Talk at Rome Meeting on Pulsars and High Energy Phenomena.

Duncan, J. C.: 1921, Proc. Nat. Acad. Sci. 7, 179.

Duncan, J. C.: 1939, Astrophys. J. 89, 482.

Gott, J. R., Gunn, J. E., and Ostriker, J. P.: 1970, Astrophys. J. 160, L91.

Lynds, R., Maran, S. P., and Trumbo, D. E.: 1969, Astrophys. J. 155, L121.

Mayall, N. U.: 1937, Publ. Astron. Soc. Pacific 49, 101.

Mayall, N. U.: 1962, Science 137, 91. 
Mihalas, D., and Routly, P.: 1968, Galactic Astronomy, W. H. Freeman \& Co., p. 114-115.

Minkowski, R.: 1968, 'Nonthermal Galactic Radio Sources' in B. Middlehurst and L. Aller (eds.), Nebulae and Interstellar Matter, Univ. of Chicago Press, p. 637ff.

Minkowski, R.: 1970, Publ. Astron. Soc. Pacific 82, 470.

Münch, G.: 1958, Rev. Mod. Phys. 30, 1042.

Münch, G., Scargle, J., and Trimble, V.: 1971, The Crab Nebula, Gordon and Breach (in preparation). O'Dell, C. R.: 1962, Astrophys. J. 136, 809.

Sanford, R. F.: 1919, Publ. Astron. Soc. Pacific 31, 108.

Schmidt, M.: 1966, in A. Blaauw and M. Schmidt (eds.), Galactic Structure, Univ. of Chicago Press, p. 528.

Trimble, V.: 1968, Astron. J. 73, 535.

Trimble, V.: 1970a, Observatory 90, 221.

Trimble, V.: 1970b, Astron. J. 75, 926.

Trimble, V. and Rees, M.: 1970, Astrophys. Letters 5, 93.

Trimble, V. and Woltjer, L.: 1971, Astrophys. J. 163, L97.

Woltjer, L.: 1958, Bull. Astron. Inst. Neth. 14, 39.

Woltjer, L.: 1970, Publ. Astron. Soc. Pacific 82, 479.

\section{Discussion (on Papers 1.1 and 1.2)}

$W$. A. Fowler: How did you calculate mass of the nebula as $10 M_{\odot}$ ? Did you use the solar abundance for oxygen?

$V$. Trimble: The amount of neutral material required to produce the observed intensity of [OI] $\lambda 6300$ radiation $\left(1.24 \times 10^{-11} \mathrm{erg} \mathrm{cm}^{-3} \mathrm{sec}^{-1}\right.$; i.e. $I([\mathrm{O} \mathrm{I}]) \approx I(\mathrm{H} \beta)$ as found by C. R. O'Dell, (Astrophys. J. 136 (1962) 809) was calculated as a function of electron temperature using formulae given by M. J. Seaton (Monthly Notices Roy. Astron. Soc. 114 (1954) 154). The electron density was estimated by assuming that atoms with I. P. $<13.6 \mathrm{eV}$ are singly (radiatively) ionized and that hydrogen and helium are collisionally ionized. Useful formulae for the latter are given, e.g., by R. A. R. Parker (Astrophys. J. 139 (1964) 208). The abundances assumed are $N_{\mathrm{He}}=N_{\mathrm{H}} ; N_{\mathrm{O}}=6 \times 10^{-4} N_{\mathrm{H}}$, as suggested by Model 2 of K. Davidson and W. Tucker (Astrophys. J. 161, 437, 1970). The distance to the nebula was taken to be $2 \mathrm{kpc}$. If it is really only $1.5 \mathrm{kpc}$, the tabulated amount of matter will produce $I(\lambda 6300) \approx 1.8 I(\mathrm{H} \beta)$. The mass required cannot be more precisely estimated because the neutral material has not yet had time to come into equilibrium with the synchrotron radiation field in the 1000 year lifetime of the nebula (Davidson and Tucker, 1970). The temperature rather reflects cooling which occurred early in the nebular expansion (when the gas was perhaps much denser) and may thus be very low.

$R$. Minkowski: We do not know the proper motion of the nebula. The basic difficulty is that there seems to be no way to find the centre of mass of the nebula. Baade's ellipse is a rough fit to the outline of the nebula, but its centre is not and is not meant to be the centre of mass.

The proper motion of the pulsar has been measured, but there is a peculiar difficulty. Results obtained by different observers agree very poorly with each other, much poorer than the measuring errors admit. For the north-following star, which has about the same brightness and nebular background, the situation is quite different. All observations agree with each other quite as well as the measuring errors admit. The obvious interpretation of the poor internal agreement of the motions of the pulsar is that the measurements are affected by the presence of variable features of the nebulosity. The prime suspect is Scargle's 'thin wisp'. The observations with the largest telescopes are least strongly affected by this systematic error, but they cannot be expected to be free of it. The best that can be done at the moment is to take the mean of all observations with the 100-inch and 200-inch telescopes by van Maanen, Baade and Trimble. The position of the pulsar in +1054 computed with this mean value for the proper motion agrees reasonably well with the position of Trimble's convergence point of the filaments.

$V$. Trimble: In regard to the proper motion of NP 0531: I am pleasantly surprised to hear that the direct measurements (at least the large telescope ones) confirm the value found indirectly using the expansion centre and elapsed time.

In regard to the proper motion of the Crab Nebula: Dr. Minkowski is absolutely correct (as usual!) in saying that our lack of knowledge of the centre of mass of the present nebula prevents our 
finding its proper motion by the method used for 0531 - dividing change in position by time elapsed since 1054. There is, however, another possible approach to the problem, which was used to get the proper motion mentioned above. There are two ways of determining the expansion centre of the nebula: (1) geometrically - that is by finding the intersection (as nearly as possible, given measuring errors and perhaps turbulence) of the proper motion vectors of individual filaments.

(2) temporally - by moving backward along the proper motion vectors by the distance that each filament would cover in a fixed increment of time until, as nearly as possible, all features meet in the same place at the same time.

If the presupernova star had no proper motion of its own, the centre thus found is the same as in the first method.

But if there is some overall proper motion, then this method will give a different convergence centre.

Notice that in this latter case, the scatter of points at the time of best convergence might be expected to be somewhat larger than if there were no overall proper motion, but the effect is small compared to the scatter caused by measuring errors in the case of the Crab Nebula - that is, the scatter is the same for methods (1) and (2). In addition, the centres found by the two methods are virtually identical - no effect of this type is found to within the uncertainty of position of the two centres. It is evident that a variety of things, including asymmetrical acceleration of the nebular expansion, could invalidate the proper motion found in this way, but not knowing the centre of mass is not, a priori, one of them, provided that enough individual proper motions are available to represent the entire nebula.

R. Minkowski: Why does the neutral gas stay in filaments?

V. Trimble: The filaments as a whole appear to be kept together by a pressure balance which involves differences in density, temperature, magnetic field, and (perhaps) relativistic particle pressure across their boundaries. The 'neutral' gas will not be exempt even from the effects of the magnetic field, because at the temperatures and densities discussed the electron density will be several to ten percent of the total particle density.

L. Aller: It is extremely difficult to deduce the chemical composition of a gaseous nebula from an emission line spectrum if the gas contains numerous filaments and 'low densations'. Among less exotic nebulae, the effects are best exhibited in NGC 7027 where the available data clearly indicated a strongly inhomogeneous structure (Aller, Astrophys. J. 120, 401, 1954). If we use density - sensitive line ratios of (S II), (OII), (CliI), ArIv), with recent cross-section calculations (Czyzak, Seaton and their associates) we find that no single choice of density and temperature can represent the data. Either the atomic parameters or observed line intensities are grossly in error (which seems unlikely) or very substantial fluctuations in $T$ and $N_{e}$ must exist. These fluctuations must be taken into account in trying to estimate chemical compositions.

J. Kristian: Have you actually seen Scargle's original plates?

$V$. Trimble: I saw a better reproduction of the late 1969 Lick plate at the Rome pulsar meeting in December 1969 and was convinced at the time that there was a 'thin wisp' in the required position, but I agree that there is some room for doubt on the basis of the pictures presented here.

D. W. Richards: What is the origin of the figure $0^{\prime \prime} .009 / \mathrm{yr}$ for proper motion of the pulsar?

$V$. Trimble: My 'favourite' proper motion for NP 0531 is derived by taking the angular separation of the present pulsar position from the nebular expansion centre (after all, everything must have been in the same place when the supernova explosion occurred) and dividing by the time elapsed since 1054 . Direct measurements of the proper motion made on plates taken with large telescopes, as discussed by R. Minkowski (Publ. Astron. Soc. Pacific 82, (1970) 470 report from the Flagstaff Conferences, June 1969),confirm it to within the errors of the observations, despite the difficulties of the measurement as outlined by him elsewhere in this discussion.

$L$. Woltjer: These agree in magnitude but not in direction.

$R$. Minkowski: They do agree if you use the best measurements.

J. E. Baldwin: The proper motion of $0^{\prime \prime} .009 / \mathrm{yr}$ corresponds to a delay in the pulsar timing measurements of about $100 \mu \mathrm{sec}$. Is this accuracy easily achievable when all the corrections have been put into the observations?

J. A. Roberts: The behaviour of the Crab Nebula pulsar is so irregular that I doubt if such an effect could be disentangled from other effects.

$P$. Horowitz: I'd like to comment on the suggestion of measuring the proper motion of the Crab pulsar from timing measurements. The component of motion along the line of sight is manifested as a 
simple doppler shift, and is therefore unmeasurable since we don't know the unshifted pulsar period. The transverse motion (proper motion) would produce a yearly sinusoid in the observed arrival times, due to parallax, of about $25 \mu \mathrm{sec}$ amplitude (if the proper motion is $0.01 \mathrm{sec} / \mathrm{yr}$ ). From our experience with optical timing measurements we can say that such variations are completely swallowed up by 'jumps' and other anomalies in the Crab pulsar period, and are therefore unmeasurable.

R. Hills: At Lick we are also making optical timing measurements and we hoping that when we have data covering about 2 years we will be able to estimate the component of the proper motion along the ecliptic. The problem is to separate out the term of one year period and find the rate of change of that term. 